\title{
Inhibition of MicroRNA-124 Reduces Cardiomyocyte Apoptosis Following Myocardial Infarction via Targeting STAT3
}

\author{
Fang He $\mathrm{He}^{\mathrm{a}}$ Huibin Liu ${ }^{\mathrm{a}}$ Jing Guo ${ }^{\mathrm{a}}$ Di Yang ${ }^{\mathrm{a}}$ Yang Yu $\mathrm{u}^{\mathrm{a}}$ Jie Yu \\ Xiuqing Yan ${ }^{\mathrm{a}}$ Juan $\mathrm{Hu}^{\mathrm{a}}$ Zhimin $\mathrm{Du}^{\mathrm{a}, \mathrm{b}}$ \\ anstitute of Clinical Pharmacy, the Second Affiliated Hospital of Harbin Medical University, The \\ University Key Laboratory of Drug Research, Heilongjiang Province, Harbin, ${ }^{b}$ Department of Clinical \\ Pharmarcology, College of Pharmacy, Harbin Medical University, Harbin, China
}

\section{Key Words}

Myocardial infarction $\cdot$ miR-124 $•$ Apoptosis $\cdot$ STAT3

\begin{abstract}
Background/Aims: MicroRNAs play an important role in regulating myocardial infarction (MI)-induced cardiac injury. MicroRNA-124 (miR-124) plays a vital role in regulating cellular proliferation, differentiation and apoptosis. Although the alteration of miR-124 was confirmed in peripheral blood of $\mathrm{MI}$ patients, little is known regarding the biological functions of miR124 in cardiomyocytes. This study was designed to explore the role of miR-124 in MI and its underlying mechanisms. Methods: Real-time PCR was used to quantify the microRNAs levels. TUNEL and Flow cytometry were performed to measure cell apoptosis. Western blot analysis was employed to detect expression of $\mathrm{BCl}-2$, Bax, Caspase- 3 and STAT3 proteins. Results: We revealed that miR-124 was significantly up-regulated in a mice model of $\mathrm{MI}$ and in neonatal rat ventricular myocytes (NRVMs) with $\mathrm{H}_{2} \mathrm{O}_{2}$ treatment. $\mathrm{H}_{2} \mathrm{O}_{2}$ treatment induced cardiomyocyte injury with reduced cell viability and enhanced apoptotic cell death, whereas silencing expression of miR-124 by AMO-124 (antisense inhibitor oligodeoxyribonucleotides) alleviated these deleterious changes. AMO-124 decreased the expression of Bax and cleavedcaspase-3 and upregulated the expression of $\mathrm{Bcl}-2$ in $\mathrm{H}_{2} \mathrm{O}_{2}$-treated NRVMs. Besides, AMO124 improved mitochondrial dysfunction of NRVMs induced by $\mathrm{H}_{2} \mathrm{O}_{2}$ treatment. Moreover, antagomir-124 markedly decreased the infarct area and apoptotic cardiomyocytes and improved cardiac function in MI mice. Furthermore, we identified STAT3 as a direct target of miR-124, and downregulation of miR-124 ameliorated the diminished levels of STAT3 and p-STAT3 (Tyr705) in response to $\mathrm{H}_{2} \mathrm{O}_{2}$ or MI. STAT3 inhibitor, stattic, was shown to attenuate the elevation of p-STAT3 in NRVMs with AMO-124 transfection. Inhibiting of STAT3 activity by stattic abrogated protective effects of AMO-124 on $\mathrm{H}_{2} \mathrm{O}_{2}$-induced cardiomyocytes apoptosis.
\end{abstract}

F. He and $\mathrm{H}$. Liu contributed equally to this work.

\begin{tabular}{ll}
\hline Zhimin Du & Inst. of Clinical Pharmacy of the Second Affiliated Hospital, Harbin Med.Univ. \\
& Xuefu Road 246, Nangang District, Harbin 150086, Heilongjiang Province (China) \\
& Tel. +86-451-86605353, E-Mail dzm1956@126.com
\end{tabular}

\section{KARGER}




\section{Cellular Physiology Cell Physiol Biochem 2018;51:186-200

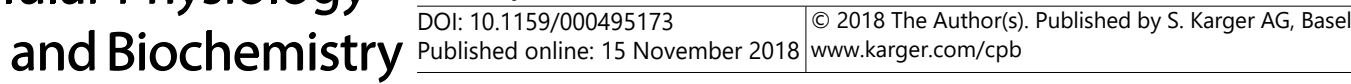 \\ He et al.: miR-124 Inhibition Reduces Cardiomyocyte Apoptosis}

Conclusion: Taken together, our data demonstrate that downregulation of miR-124 inhibits MI-induced apoptosis through upregulating STAT3, which suggests the therapeutic potential of miR-124 for myocardial infarction.

(C) 2018 The Author(s)

Published by S. Karger AG, Basel

\section{Introduction}

Acute myocardial infarction (AMI), a condition in which myocardial perfusion is arrested, is one of the leading causes of morbidity and mortality worldwide [1]. Myocardial apoptosis is a major type of cardiomyocyte death during MI, and the surviving cardiomyocytes undergo additional progressive loss, which finally develops into heart failure. Therefore, inhibition of myocardial apoptosis is an appealing therapeutic strategy for improving cardiac function, preventing ventricular remodeling and decreasing the incidence of cardiovascular events after MI [2]. However, the cellular mechanisms underlying ischemia-induced apoptosis in cardiomyocytes are complicated and poorly understood.

MicroRNAs (miRNAs) are a class of small, endogenous and non-encoding RNA molecules with about 18-22 nucleotides that mediate posttranscriptional gene silencing by inhibiting mRNA translation or cleaving the target mRNA. Growing evidence has shown that miRNAs are involved in the pathogenesis of cardiovascular diseases, including cardiac injury $[3,4]$, arrhythmia [5], cardiac hypertrophy [6] and heart failure [7] and so on. Clinical research and animal experiments indicate that miRNAs are potential biomarkers and therapeutic targets for cardiac ischemia $[8,9]$.

MiR-124 was firstly reported being abundantly expressed in neuronal cells [10]. Furthermore, the critical roles of miR-124 in the occurrence and progression of various diseases are being increasingly recognized. Several studies have suggested that miR124 is significantly downregulated in several human cancers, such as gastric cancer [11], hepatocellular carcinoma [12], colorectal cancer [13] and cervical cancer [14]. In contrary, upregulation of miR-124 suppresses cell proliferation and promotes apoptosis $[10,15]$. Moreover, STAT3, a key cellular survival factor, has been shown to be an important target of miR-124 and upregulates several proteins which are responsible for suppressing cell apoptosis and inducing tumor growth $[10,15]$. In addition, it has been shown that miR124 downregulated STAT3 expression directly in hippocampal neurons and bone marrow derived mesenchymal stem cells $[16,17]$. Previous study uncovered the important role of miR-124 in cardiovascular diseases including myocardial remodeling [18]. The expression of miR-124 in AMI and its significance, however, has not been fully illustrated. Thus, there is a possibility that miR-124 might contribute to MI though regulating the expression of STAT3. In this study, we firstly tested the changes of miR-124 expression in both cellular and mouse model of MI. We revealed that miR-124 was significantly upregulated in these two models and inhibition of miR-124 decreased apoptosis and preserved cell survival. Thus, the downregulation of miR-124 may provide a therapeutic target for MI in mice.

\section{Materials and Methods}

\section{Animals}

Healthy adult male Kunming mice (25-30 g) used in the present study were kept under standard animal room conditions (temperature, $23 \pm 1^{\circ} \mathrm{C}$; humidity, $55 \pm 5 \%$ ) with food and water ad libitum for 1 week before the experiments. All experimental protocols were done in accordance with the Institutional Animal Care and Use Committee of the Harbin Medical University and the Ethic Committee of Harbin Medical University, PR China. 


\section{Cellular Physiology Cell Physiol Biochem 2018;51:186-200

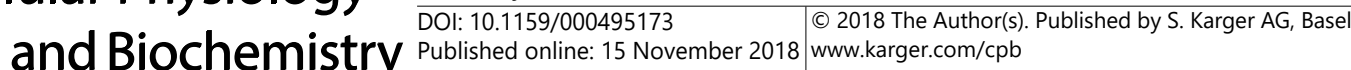

He et al.: miR-124 Inhibition Reduces Cardiomyocyte Apoptosis

\section{Neonatal rat ventricular myocytes}

Primary neonatal rat myocytes were isolated from newborn Sprague-Dawley rat heart ventricles by trypsin solution. Briefly, hearts of newborn SD rats were removed aseptically after the rats were decapitated. And then the hearts were minced in serum-free Dulbecco's Modified Eagle Media (DMEM, HyClone, UT) and digested in $0.25 \%$ trypsin solution. The cell suspension was centrifuged at $2500 \mathrm{rpm}$ for $3 \mathrm{~min}$ and resuspended in DMEM medium containing $10 \%$ fetal bovine serum. Then the non-adherent cardiomyocytes were plated into culture flasks (noncoated). Cardiomyocytes were cultured under a condition of $5 \% \mathrm{CO}_{2}$ at $37^{\circ} \mathrm{C}$.

\section{MiRNAs transfection}

MiR-124, AMO-124 and negative control (NC) were synthesized by Guangzhou RiboBio (Guangzhou, China). AMO-124 contained 2'-0-methyl modifications. After starvation in serum-free medium for $12 \mathrm{~h}$, cardiomyocytes were transfected with X-treme GENE siRNA transfection reagent (Roche, Germany) according to the manufacturer's instructions. After transfection for $48 \mathrm{~h}$, NRVMs were treated with $\mathrm{H}_{2} \mathrm{O}_{2}$ $(200 \mu \mathrm{M})$ and incubated for $4 \mathrm{~h}$.

\section{Real-time RT-PCR}

For reverse transcription PCR (RT-PCR), total RNA was isolated from heart tissue or cultured NRVMs using TRIzol reagent (Invitrogen, CA) following the manufacturer's instructions. The levels of miR-124 were determined using SYBR Green incorporation on Roche Light-Cycler480 Real Time PCR system (Roche, Germany), with U6 as the internal control. The sequences of primers were: miR124 forward: 5'-TAAGGCACGCGGTGAATGCC-3' reverse: $5^{\prime}$-CAGTGCGTGTCGTGGAGT-3'; U6 forward: 5'-GCTTCGGCACATATACTAAAAT-3' and reverse: 5'-CGCTTCACGAATTTGCGTGTCAT-3'; Relative miR-124 level was calculated based on the threshold cycle values and normalized to the internal U6.

\section{TUNEL Staining}

The In situ Cell Death Detection Kit (TUNEL fluorescence FITC kit, Roche, Germany) was used to label apoptotic cells according to the manufacturer's instruction. After TUNEL staining, the heart sections (3 days post-MI) or cardiomyocytes ( $4 \mathrm{~h}$ after $\mathrm{H}_{2} \mathrm{O}_{2}$ treated) were immerged into the Hoechst 33342 (Sigma-Aldrich, USA) solution to stain nuclei of living and apoptotic cells. Fluorescence staining was viewed by a laser scanning confocal microscope (Olympus, Fluoview1000, Japan). The numbers of total cells and TUNELpositive cells were counted by Image-Pro plus version.

\section{MTT Assay}

Cultured NRVMs were plated in 96-well plates and cell viability was evaluated by 3-(4,5-dimethylthiazol2-yl)-2, 5-diphenyltetrazolium bromide (MTT) assay according to the manufacturer's instructions. After miRNA transfection and $\mathrm{H}_{2} \mathrm{O}_{2}$ treatment, $20 \mu \mathrm{L}$ MTT $(0.5 \mathrm{mg} / \mathrm{mL}$; Sigma-Aldrich, USA) was added to each well, and incubated with the cells for $4 \mathrm{~h}$ at $37^{\circ} \mathrm{C}$. And then $150 \mu \mathrm{L}$ DMSO was used to dissolve the cell layer. The absorbance was measured by spectrophotometer (Tecan, Austria) at $490 \mathrm{~nm}$.

\section{Western blot analysis}

Total protein was extracted from the heart tissue or the cultured NRVMs. Briefly, protein samples (80 $\mu \mathrm{g})$ were fractionated by SDS-PAGE (10\% polyacrylamide gels) and blotted to nitrocellulose membrane, which was blocked with $5 \%$ non-fat milk for $2 \mathrm{~h}$ at room temperature and then incubated at $4{ }^{\circ} \mathrm{C}$ overnight with the following primary antibodies: STAT3 (Cell Signaling Technology, USA), p-STAT3 (phospho-STAT3 at Tyr 705; Cell Signaling Technology, USA); Bcl-2 (Cell Signaling Technology, USA), Bax (Proteintech, China) and $\beta$-actin (ZSGB-Bio, China). Specifically, a caspase- 3 antibody (Cell Signaling Technology, USA) was used to recognize full-length caspase-3 (35 kDa) and caspase-3 fragment (17 kDa) resulting from cleavage at aspartate position175. The membranes were washed with PBS-T, the membrane was incubated with secondary antibody for $1 \mathrm{~h}$. Western blot bands were quantified by Odyssey CLx v2.1 software (LI-COR Biosciences, USA). 


\section{Cellular Physiology Cell Physiol Biochem 2018;51:186-200 and Biochemistry \begin{tabular}{l|l} 
DOI: 10.1159/000495173 & (c) 2018 The Author(s). Published by S. Karger AG, Basel \\
www.karger.com/cpb
\end{tabular}}

He et al.: miR-124 Inhibition Reduces Cardiomyocyte Apoptosis

Detection of apoptosis by flow cytometry (FCM)

Apoptosis was quantified using an Annexin V-FITC/ propidium iodide (AV/PI) apoptosis kit. The staining procedures were performed in accordance with the manufacturers' instructions. After treatment, cells were collected and washed twice with cold PBS and then stained with Annexin V-FITC and PI respectively for $10 \mathrm{~min}$ in the dark at RT. FITC or PI positive cells were determined by flow cytometry (BD Bioscience, USA).

Administration of antagomiR-124 and animal model of $M I$

Adult male mice (25-30g) were randomly divided into three groups: sham, MI (infarction for three days) and MI+antagomir-124. Antagomir-124, a construct with chemically modification and with cholesterol moiety conjugation, was used to inhibit miR-124 expression in vivo. A bulldog clamp was used to incarcerate the aorta and then antagomir-124 was injected into the left ventricle myocardium. Mice were subjected to the ligation of the mid-left anterior descending (LAD) artery to induce MI. Briefly, animals were anesthetized by intraperitoneal injection of affinity $(0.01 \mathrm{~mL} / \mathrm{g})$. The LAD artery was ligated by using $8 / 0$ suture after the heart was exposed by a thoracotomy. And then the chest cavity was sutured by 5-0 suture. Sham-operated mice underwent an identical procedure except that the suture was passed around the vessel without LAD occlusion.

Measurement of infarct size

Three days after MI, the left ventricle was cut into 2-mm thick slices and stained with $1 \%$ triphenyltetrazolium chloride (TTC, Sigma-Aldrich, USA) for $20 \mathrm{~min}$ at $37^{\circ} \mathrm{C}$. The infarct area was identified by stainless region while the live area turned red. The infarct area (IA) was calculated using Image ProPlus 5.0 software (Media Cybernetics, UK).

\section{Echocardiographic measurements}

Echocardiography was performed after the LAD ligation for three days by using an ultrasound machine (Panoview 31500 , Cold Spring Biotech, Taiwan, China) equipped with a 30-MHz phased-array transducer. Left ventricular end-diastolic diameter and end-systolic diameter were measured by M-mode tracings and the percentage of ejection fraction (EF\%) and fractional shortening (FS\%) of left ventricular were calculated.

\section{Determinations of LDH and Caspase-3 activity}

The activities of LDH in serum and Caspase-3 in heart tissue were measured to detect the degree of cardiac injury. LDH (Jiancheng Biotechnology Co., China) and Caspase-3 (Beyotime, China) activities were detected using commercial kits according to the manufacturers' instructions.

\section{Statistical analysis}

All data were presented as mean \pm SEM and analyzed by SigmaPlot and SigmaStat Software (Jandel Scientific, USA). Paired t test or Student's t test was used where appropriate. A two-tailed $P<0.05$ was considered to be statistically significant.

\section{Results}

miR-124 expression is upregulated in response to $\mathrm{MI}$ or $\mathrm{H}_{2} \mathrm{O}_{2}$

In this study, we first examined the expression level of miR-124 in different zones of the heart after MI for $4 \mathrm{~h}, 24 \mathrm{~h}$ and $72 \mathrm{~h}$, respectively. Real-time quantitative PCR analysis revealed that miR-124 expression was increased in infarcted and border zones (Fig. 1A and 1B) but not in the remote zone of mouse ventricles (Fig. 1C). Furthermore, cardiomyocytes were subjected to $\mathrm{H}_{2} \mathrm{O}_{2}$ to simulate cardiomyocyte injury model in vitro. Cell viability was markedly decreased by $\mathrm{H}_{2} \mathrm{O}_{2}$ treatment in a concentration and time-dependent manner as shown in previous study $[19,20]$. Here, $200 \mu \mathrm{M} \mathrm{H}_{2} \mathrm{O}_{2}$ treated for $4 \mathrm{~h}$ was chosen to stimulate apoptosis in subsequent in vitro experiments. Consistently, we found that the miR-124 level was significantly higher in $\mathrm{H}_{2} \mathrm{O}_{2}$-treated cardiomyocytes compared with control cells (Fig. 1D). Then, miR-124 expression was downregulated or upregulated in cardiomyocytes by 


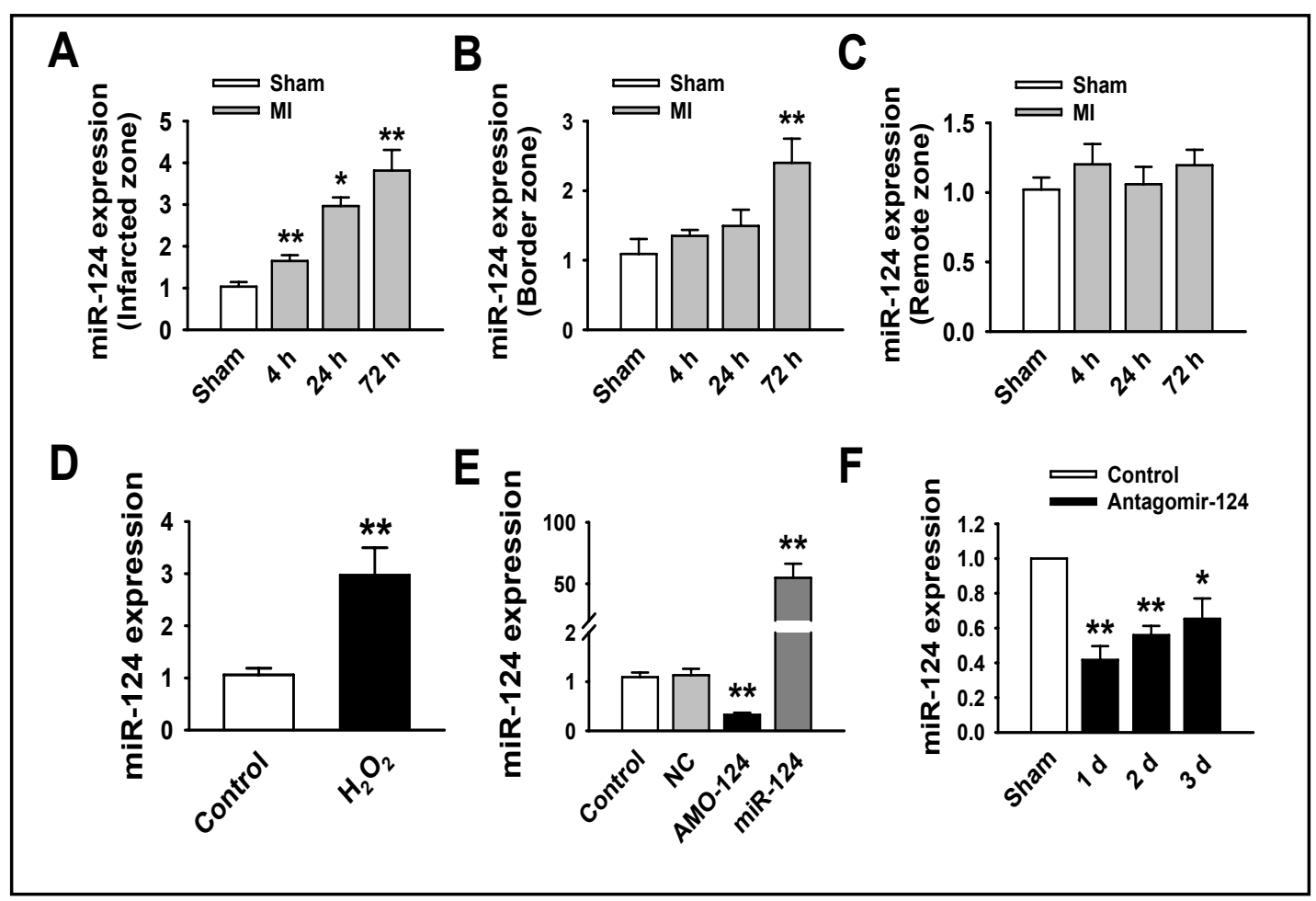

Fig. 1. miR-124 is upregulated in infarcted myocardium and cardiomyocytes treated with $\mathrm{H}_{2} \mathrm{O}_{2}$ ( $(A)$ and (B) Real-time PCR analysis indicates that miR-124 was increased in the infarcted and border zones of MI mice compared with sham mice. $\mathrm{n}=6 .{ }^{*} \mathrm{P}<0.05,{ }^{* *} \mathrm{P}<0.01$ vs. Sham group. (C) miR-124 level in remote zone of mouse ventricles. $n=6$. (D) miR-124 is increased in cardiomyocytes treated with $\mathrm{H}_{2} \mathrm{O}_{2}(200 \mu \mathrm{M})$ for $4 \mathrm{~h}$. $\mathrm{n}$ $=6 .{ }^{*} \mathrm{P}<0.05,{ }^{* *} \mathrm{P}<0.01$ versus Control group. (E) miR-124 level was obviously decreased in NRVMs after transfecting with AMO-124 and dramatically increased after transfecting with miR-124 compared with negative control (NC). $\mathrm{n}=6$. ${ }^{* *} \mathrm{P}<0.01$ vs. NC group. (F) miR-124 expression in heart tissue was dramatically decreased in antagomiR-124 group compared with NC group. $\mathrm{n}=6 .{ }^{*} \mathrm{P}<0.05,{ }^{* *} \mathrm{P}<0.01$ vs. NC group.

AM0-124 or miR-124 transfection. Real-time PCR results showed that miR-124 expression level markedly decreased in AMO-124 transfection group compared with negative control (NC) transfection group (Fig. 1E). Conversely, miR-124 was significantly increased in miR124 transfection group (Fig. 1E). Moreover, antagomir-124 was injected in mice heart using our delivery method to downregulate miR-124 level in vivo. As shown in Fig. 1F, 1, 2 and 3 days after injection of antagomir-124, miR-124 expression was markedly decreased in the heart tissue.

Downregulation of miR-124 inhibits cardiomyocyte apoptosis induced by $\mathrm{H}_{2} \mathrm{O}_{2}$

Next, we modulated miR-124 expression to determine its role in regulating cardiomyocyte apoptosis in response to $\mathrm{H}_{2} \mathrm{O}_{2}$. TUNEL-stained photomicrographs of cardiomyocytes transfected with NC, miR-124, and AMO-124 are shown in Fig. 2A. The results suggested that the silencing of miR-124 by its antisense oligonucleotides (AMO-124) protected cardiomyocyte from $\mathrm{H}_{2} \mathrm{O}_{2}$-induced apoptosis, while co-transfected with miR-124 abrogates the beneficial effect of AMO-124 (Fig. 2B). MTT assay showed that $\mathrm{H}_{2} \mathrm{O}_{2}(200 \mu \mathrm{M})$ reduced cell viability and AMO-124 significantly alleviated this detrimental change (Fig. 2C). Furthermore, flow cytometry was used to determine the impact of miR-124 on $\mathrm{H}_{2} \mathrm{O}_{2}$-induced cardiomyocyte apoptosis. We found that the apoptotic percentage of cardiomyocytes was increased by $\mathrm{H}_{2} \mathrm{O}_{2}$ treatment, which was significantly diminished by AMO-124 (Fig. 2D and 2E). Therefore, these results proved that silencing of miR-124 prevented $\mathrm{H}_{2} \mathrm{O}_{2}$-induced cardiomyocyte apoptosis and promoted cell survival. 


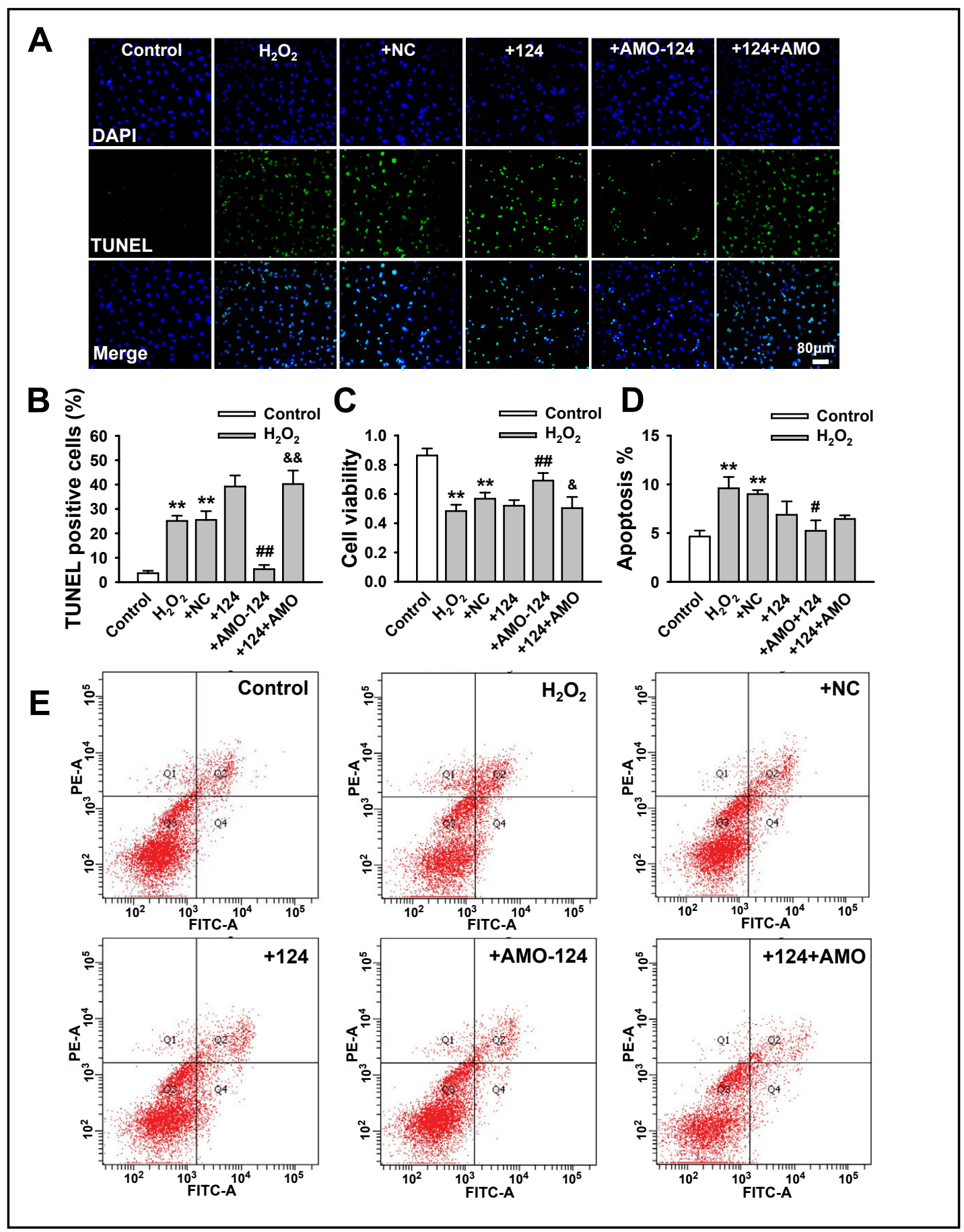

Fig. 2. AMO-124 attenuates $\mathrm{H}_{2} \mathrm{O}_{2}$-induced cardiomyocyte apoptosis. (A) Representative images of TUNEL staining of cardiomyocyte showed the apoptotic cells (apoptotic cells stained in green and nucleus stained in blue with DAPI). (B) Statistical analysis of TUNEL-positive cells per field indicated that AMO124 suppressed $\mathrm{H}_{2} \mathrm{O}_{2}$ treatment-induced cell apoptosis. $\mathrm{n}=6$. (C) MTT assay suggested that AMO-124 restored cell viability after $\mathrm{H}_{2} \mathrm{O}_{2}$ treatment. $\mathrm{n}=8$. (D) The quantitative presentation of apoptotic cells by Annexin V-FITC/propidium iodide (PI) staining. $n=4$. (E) The representative images of flow cytometry using Annexin V-FITC and PI staining. ${ }^{* *} \mathrm{P}<0.01$ versus Control group; ${ }^{\#} \mathrm{P}<0.05,{ }^{\# \#} \mathrm{P}<0.01$ versus NC group; ${ }^{\&} \mathrm{P}<0.05$, \&\& $\mathrm{P}<0.01$ versus +AMO-124 group. +NC, $\mathrm{H}_{2} \mathrm{O}_{2}$ +negtive control; +124, $\mathrm{H}_{2} \mathrm{O}_{2}+\mathrm{miR}-124 ;+\mathrm{AMO}-124$, $\mathrm{H}_{2} \mathrm{O}_{2}+\mathrm{AMO}-124 ;+124+\mathrm{AMO}, \mathrm{H}_{2} \mathrm{O}_{2}+\mathrm{miR}-124+\mathrm{AMO}-124$. 


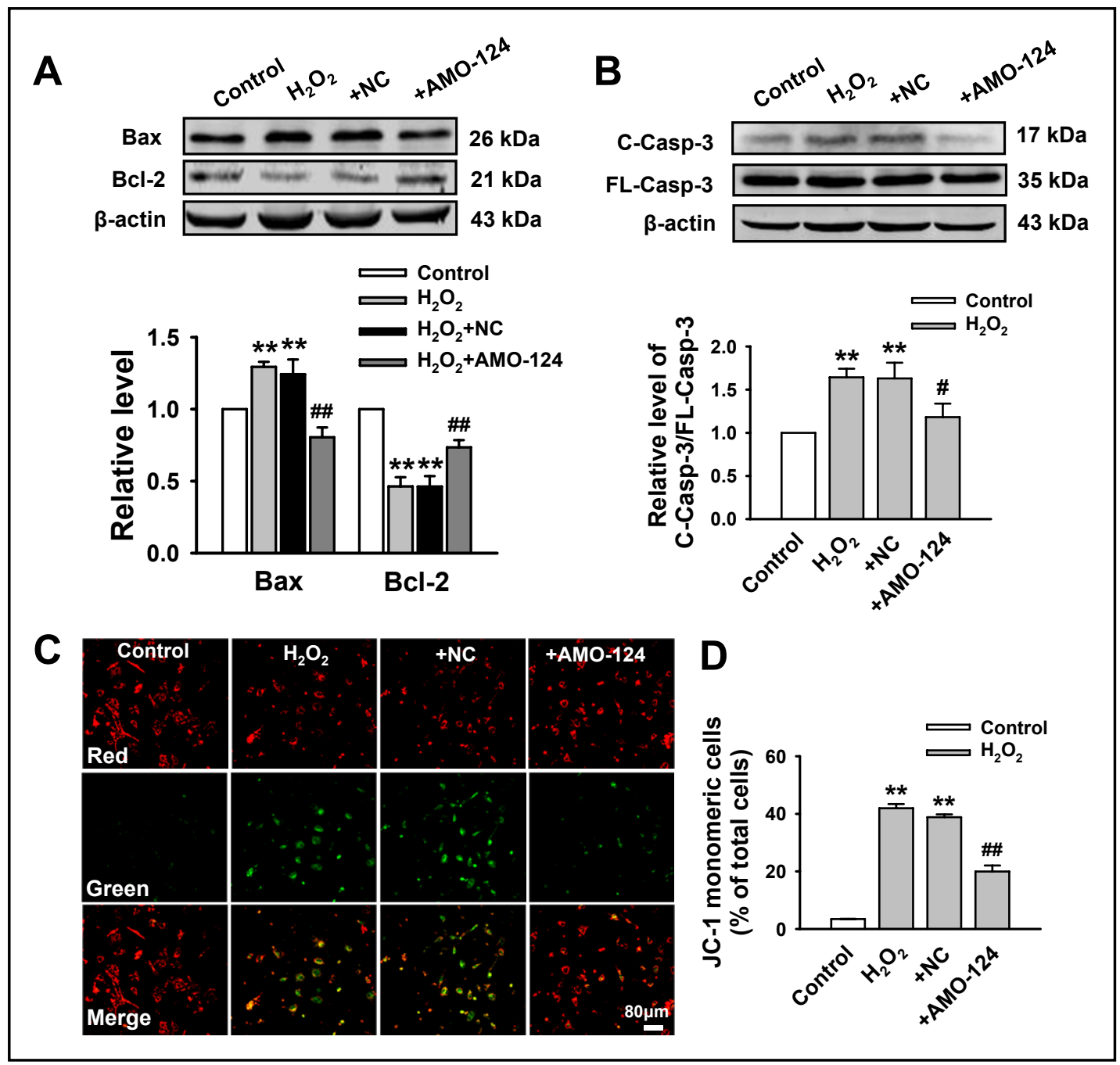

Fig. 3. Effects of miR-124 on apoptosis-related proteins and mitochondrial membrane potential of NRVMs. (A) The expression of Bax and Bcl- 2 was determined by Western blot analysis. $\mathrm{n}=5$. ${ }^{* *} \mathrm{P}<0.01$ versus Control group; ${ }^{\# P} \mathrm{P}<0.01$ versus +NC group. (B) The expression of cleaved-caspase-3 (C-Casp-3) and full length caspase-3 (FL-Casp-3) was determined by Western blot analysis and the relative ratio of C-Casp-3 and FLCasp-3 protein levels was calculated. $\mathrm{n}=5$. ${ }^{* *} \mathrm{P}<0.01$ versus Control group; ${ }^{\#} \mathrm{P}<0.05$ versus $+\mathrm{NC}$ group. (C) Representative images of JC-1 fluorescence. $\Delta \psi \mathrm{m}$ was assessed by confocal microscopy using JC-1 probe in NRVMs. (D) Quantitative analysis of fluorescence intensity in NRVMs of (C). Data was normalized as the ratio of green fluorescence to total (red plus green) fluorescence. $\mathrm{n}=5$. ${ }^{* *} \mathrm{P}<0.01$ versus Control group; ${ }^{\# \#} \mathrm{P}<0.01$ versus +NC group. $+\mathrm{NC}, \mathrm{H}_{2} \mathrm{O}_{2}+$ negtive control; +AMO-124, $\mathrm{H}_{2} \mathrm{O}_{2}+\mathrm{AMO}-124$.

miR-124 regulates apoptosis related signal proteins and mitochondrial membrane potential in NRVMS

We further investigated if the silencing of miR-124 exerts significant influence on expression of Bcl-2, Bax and Caspase- 3 proteins in cultured NRVMs exposed to $\mathrm{H}_{2} \mathrm{O}_{2}$. As shown in Fig. 3A, $\mathrm{H}_{2} \mathrm{O}_{2}$ remarkably improved the expression of Bax but suppressed Bcl-2 expression. On the contrary, AMO-124 could reverse above alterations. We noted that $\mathrm{H}_{2} \mathrm{O}_{2}$ increased the level of cleaved caspase-3, an active form of caspase-3, which was diminished by miR-124 downregulation (Fig. 3B). 
Next, we examined the effect of miR-124 on the mitochondrial membrane potential $(\Delta \psi \mathrm{m})$. JC- 1 is a fluorescent probe that applied widely to detect the $\Delta \psi \mathrm{m}$. When the $\Delta \psi \mathrm{m}$ is high, JC-1 aggregates into a polymer to generate a red fluorescence in the mitochondrial matrix. When the $\Delta \psi \mathrm{m}$ is low, JC-1 produces a green fluorescence without aggregating. In this study, the relative proportion of green to total (red plus green) fluorescence under confocal microscopy was used to assess $\Delta \psi \mathrm{m}$. As shown in Fig. 3C and 3D, miR-124 silencing by its AMO significantly reduced the $\quad \mathrm{H}_{2} \mathrm{O}_{2}$-induced downregulation of $\Delta \psi \mathrm{m}$. In total, these results revealed that miR124 downregulation inhibited apoptosis of cardiomyocytes by preventing m i t o c h o n d rial dependent pathway of

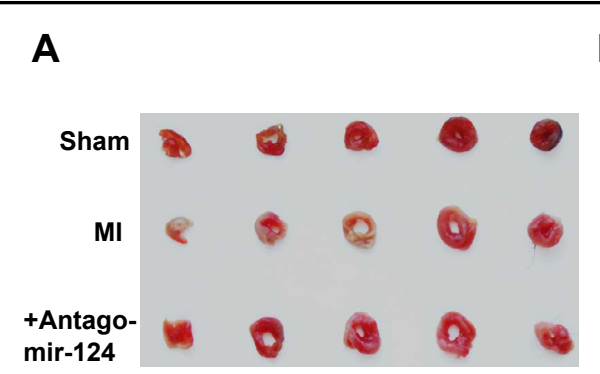

\section{B}

C

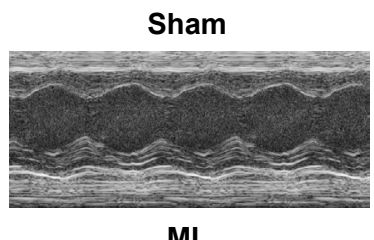

MI

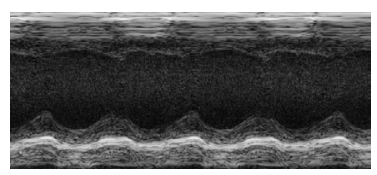

MI + Antagomir-124

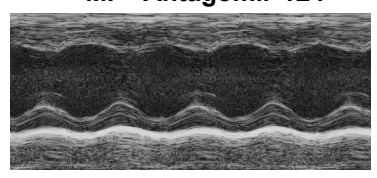

D

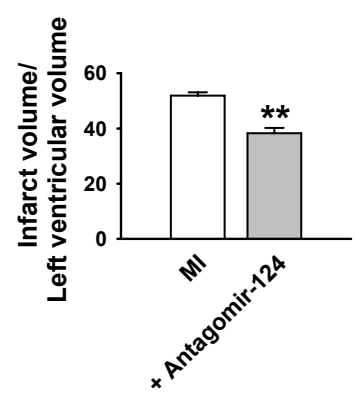

$\mathbf{E}$
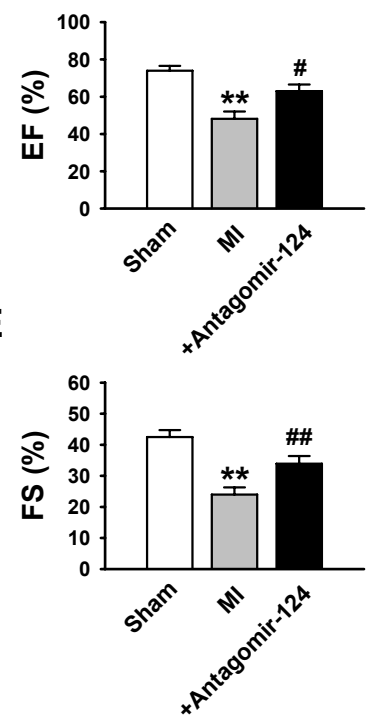

Fig. 4. Reduction of infarct size and improvement of cardiac function by antagomir-124. (A) Representative images showed infarct areas in cross section slices. (B) Statistical analysis of infarct volume/left ventricular volume for infarct areas detection. $\mathrm{n}=5$. ${ }^{* *} \mathrm{P}<0.01$ versus MI group; (C) Representative photographs of heart function. (D) Ejection fractions (EF\%). $\mathrm{n}=6$. (E) Fractional shortening (FS\%). $\mathrm{n}=6 .{ }^{* *} \mathrm{P}<0.01$ versus sham group; ${ }^{\#} \mathrm{P}<0.05,{ }^{\# \#} \mathrm{P}<0.01$ versus MI group. +antagomir-124, MI+antagomir-124. apoptosis.

Downregulation of miR-124 attenuates infarct size and improves cardiac function of MI mice

We established the mice model of myocardial infarction and examined the effect of downregulation of miR-124 on MI injury. We administered the antagomir-124 into the left ventricle of mice before coronary artery ligation and measured the infarct size and cardiac function three days after MI. The infarct size was evaluated by TTC staining. Fig. 4A shows that significant decreased myocardial infarct size was observed in the antagomir-124 pretreatment group relative to MI group (Fig. 4B). Moreover, Fig. 4C-4E showed that in vivo deliver of antagomir-124 into the myocardium markedly attenuated MI-induced cardiac dysfunction. In addition, MI markedly decreased ejection fraction (EF\%) and fractional shortening (FS\%) values in mice hearts (Fig. 4C-4E). In contrast, the values of EF\% and FS\% in antagomir-124 transfected hearts were significantly greater than in the un-transfected group after MI (Fig. 4C-4E). 


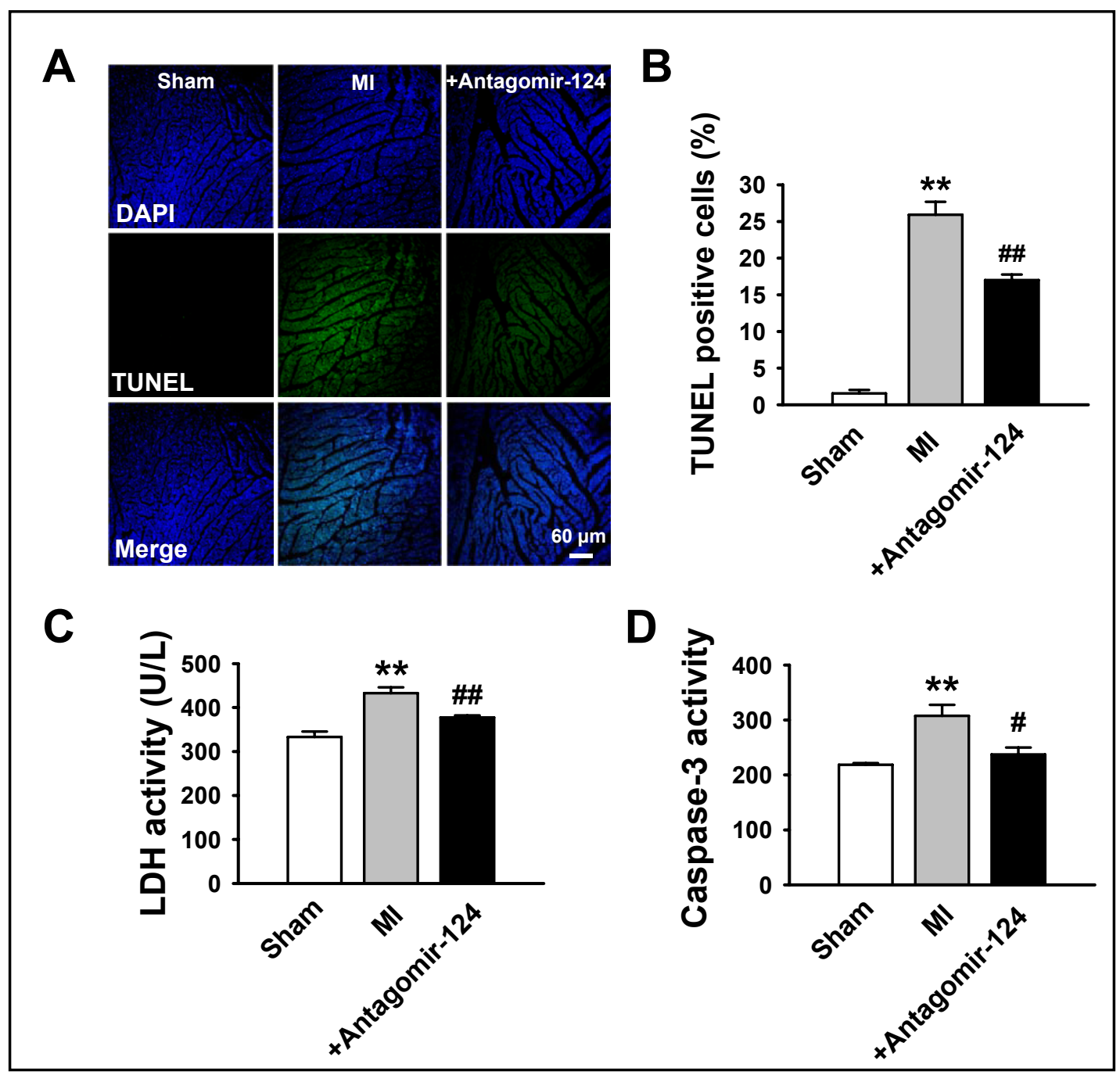

Fig. 5. Anti-apoptotic effect of antagomir-124 in the mouse model of MI. (A) Effects of antagomir-124 on ischemia-induced apoptosis were evaluated by TUNEL assay. (B) TUNEL positive cells (\%). $n=8$. (C) Serum lactate dehydrogenase (LDH) activity was measured in MI and antagomiR-124 injection mice. $n=4$. (D) Caspase- 3 activity was measured in heart tissue of MI mice. $\mathrm{n}=5$. ${ }^{*} \mathrm{P}<0.01$, versus sham group; ${ }^{\#} \mathrm{P}<0.05$, \#\# $\mathrm{P}<0.01$ versus MI group. +antagomir-124, MI+antagomir-124.

Downregulation of miR-124 decreases the ischemia-induced cardiomyocyte apoptosis

We then examined the effect of miR-124 on MI-induced myocardial apoptosis. As shown in Fig. 5A, myocardial apoptosis as evidenced by TUNEL positive staining was more observed in infarcted mice compared with sham control. In antagomir-124 transfected hearts, the TUNEL-positive apoptotic cells were significantly reduced compared with MI group (Fig. 5B). MI also induced increases in LDH and caspase- 3 activities compared with the sham control (Fig. 5C and 5D). In contrary, LDH and caspase-3 activities were reduced in antagomir-124 transfection group compared with MI group (Fig. 5C and 5D).

Activation of STAT3 is involved in miR-124 downregulation-mediated protection of cardiomyocyte apoptosis

As a key cellular survival factor, signal transducer and activator of transcription-3 (STAT3) plays crucial antiapoptotic roles in response to oxidative stress [21]. Furthermore, STAT3 has been identified to be a direct target of miR-124 during myogenic differentiation of bone marrow derived mesenchymal stem cells [17] and T cell-mediated immune clearance of 


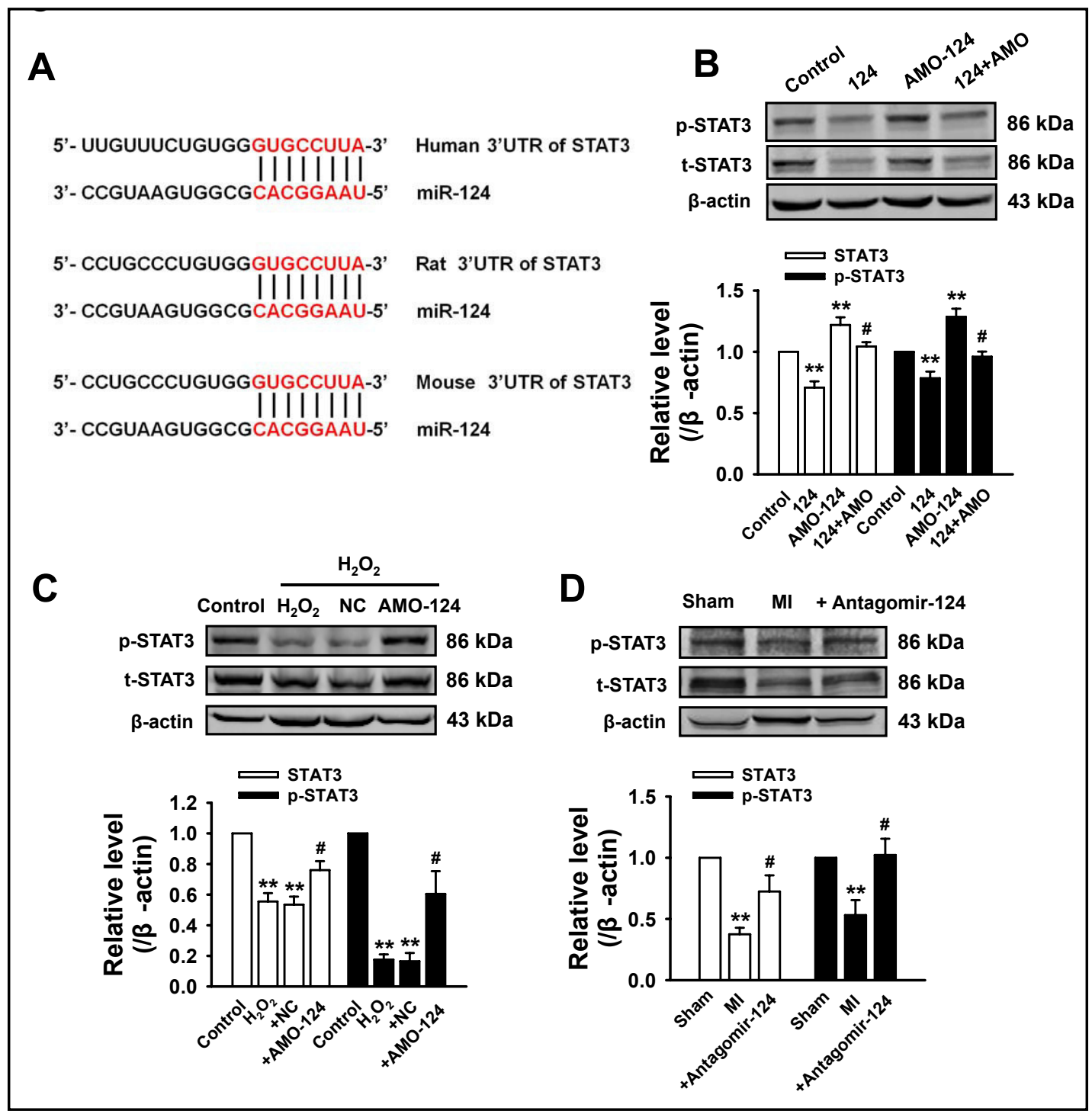

Fig. 6. STAT3 as a target gene for miR-124 in cardiomyocytes. (A) The predicted binding site of miR-124 on the 3'UTR of STAT3 gene. (B) Western blot analysis of t-STAT3 and p-STAT3 expression in NRVMs. ${ }^{* * P}<0.01$ versus Control group; "P<0.05 versus AM0-124 group. (C) Silencing of miR-124 significantly inhibited $\mathrm{H}_{2} \mathrm{O}_{2}$-induced downregulation of STAT3 activity. ${ }^{* *} \mathrm{P}<0.01$ versus Control group; ${ }^{\#} \mathrm{P}<0.05$ versus NC group. (D) Silencing of miR-124 significantly inhibited MI-induced downregulation of STAT3 activity. ${ }^{* *} \mathrm{P}<0.01$ versus Sham group; ${ }^{~} \mathrm{P}<0.05$, versus MI group. NC, negative control; 124, miR-124; 124+AMO-124, miR124+AM0-124; t-STAT3, total STAT3; p-STAT3, phosphorylated STAT3 (Y705).

glioma [10]. However, the relationship between miR-124 and STAT3 has not been validated in cardiomyocytes. At first, we analyzed the potential interaction between miR-124 and STAT3 by TargetScan target prediction software (http://www.targetscan.org/) and one miR-124-binding site was identified within the 3'-UTR of STAT3 mRNA (Fig. 6A). To further verify STAT3 as the target gene of miR-124 in cardiac myocytes, both gain-of-function and loss-of-function approaches were applied. As shown in Fig. 6B, AMO-124 increased, whereas miR-124 decreased the expressions of total STAT3 (t-STAT3) and phosphorylation of STAT3 (p-STAT3) at Tyr705 (indicating activation) in cultured cardiac myocytes. These results suggested that STAT3 may be a target gene of miR-124 in cardiomyocytes. Then, we test whether STAT3 is also involved in $\mathrm{H}_{2} \mathrm{O}_{2}$-induced cardiomyocytes apoptosis. To confirm this, we transfected cardiac myocytes with either NC or AMO-124 and then treated with $\mathrm{H}_{2} \mathrm{O}_{2}$ 


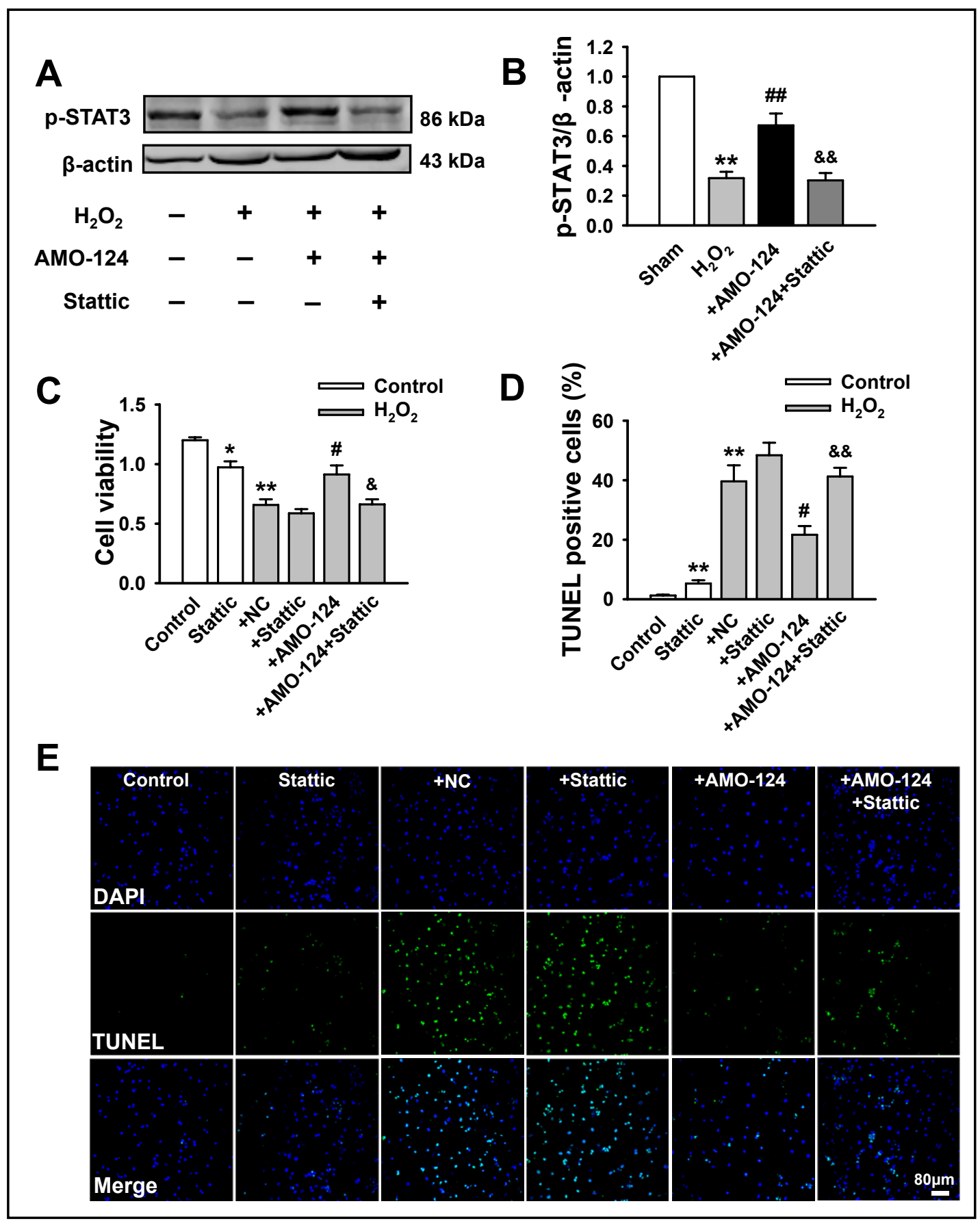

Fig. 7. STAT3 inhibition abolishes protective effect of miR-124 silencing on cardiomyocytes. (A) and (B) AMO-124 or/and STAT3 inhibitor, stattic, were used to treat NRVMs following $\mathrm{H}_{2} \mathrm{O}_{2}$ treatment and the expressions of $\mathrm{p}$-STAT3 were determined by Western blot analysis. $\mathrm{n}=5$. ${ }^{* *} \mathrm{P}<0.01$ versus Control; ${ }^{\# \#} \mathrm{P}<0.01$ versus $\mathrm{H}_{2} \mathrm{O}_{2}$ group; ${ }^{\&} \mathrm{P}<0.01$ versus $+\mathrm{AMO}-124$ group. (C) MTT assay was used to examine the effect of stattic on cell viability in the presence of $A M 0-124 . \mathrm{n}=8$. ${ }^{*} \mathrm{P}<0.05,{ }^{* *} \mathrm{P}<0.01$ versus Control group; ${ }^{\#} \mathrm{P}<0.05$ versus $+\mathrm{NC}$ group; ${ }^{\&} \mathrm{P}<0.05$ versus $+\mathrm{AMO}-124$ group. (D) TUNEL assay was used to examine the effect of stattic on $\mathrm{H}_{2} \mathrm{O}_{2}$-induced apoptosis in the presence of AMO-124. Results showed that stattic could counteract the protective effect of AMO-124 on apoptosis induced by $\mathrm{H}_{2} \mathrm{O}_{2} . \mathrm{n}=5$. ${ }^{* *} \mathrm{P}<0.01$ versus Control group; ${ }^{\mathrm{P}<0.05}$ versus $+\mathrm{NC}$ group; ${ }^{\&} \&<<0.05$ versus $+\mathrm{AMO}-124$ group. $+\mathrm{NC}, \mathrm{H}_{2} \mathrm{O}_{2}+$ negtive control; + Stattic, $\mathrm{H}_{2} \mathrm{O}_{2}+$ Stattic; +AMO-124, $\mathrm{H}_{2} \mathrm{O}_{2}+$ AMO-124; +AMO-124+Stattic, $\mathrm{H}_{2} \mathrm{O}_{2}+$ AM0-124+Stattic. 
for $4 \mathrm{~h}$ and the protein level of p-STAT3 and t-STAT3 were determined. As shown in Fig. 6C, $\mathrm{H}_{2} \mathrm{O}_{2}$ decreased both p-STAT3 and t-STAT3 protein levels. Compared with the NC, AMO-124 significantly increased the protein level of p-STAT3 and t-STAT3 (Fig. 6C). Moreover, there was a significant decrease of p-STAT3 expression in MI group (Fig. 6D), whereas antagomir-124 transfection group showed a increased expression of p-STAT3. Thus, these results reflected that the inhibition of miR-124 significantly elevated the downregulation of STAT3 activity in cardiomyocytes in response to ischemia injury.

STAT3 inhibition abolishes protective effect of miR-124 downregulation on cardiomyocytes apoptosis

To confirm the functional involvement of STAT3 in the regulation of cardiomyocytes apoptosis by miR-124, STAT3 inhibitor, Stattic was used to block the activation of STAT3 in this study. We found that Stattic significantly inhibited AM0-124 induced activity of STAT3 evidenced by dephosphorylation of $\mathrm{Y}^{705}$ in $\mathrm{H}_{2} \mathrm{O}_{2}$-treated NRVMs (Fig. 7A and 7B). Furthermore, as shown in Fig. 7C, AMO-124-mediated elevation of cell viability of $\mathrm{H}_{2} \mathrm{O}_{2}$ treated NRVMs was inhibited by Stattic. In addition, transfection of AMO-124 markedly decreased the cardiomyocytes apoptosis and this effect was alleviated by Stattic (Fig. 7D and 7E). Representative TUNEL-stained photomicrographs from cardiac myocytes with different treatment were displayed in Fig. 7E. Therefore, these data suggested that STAT3 inactivation is involved in miR-124-mediated apoptosis in cardiomyocytes.

\section{Discussion}

In the current study, our results indicate that 1) in a mice model of MI, downregulation of miR-124 protects the heart against MI injury as evidenced by its improving cardiac function, reducing the MI size and decreasing myocardial apoptosis 2) and in vitro downregulation of miR-124 significantly prevents oxidative stress-induced apoptosis and attenuating mitochondrial dysfunction through targeting STAT3.

MicroRNAs act as a sort of small and noncoding nucleotide RNAs, which negatively regulate gene expression by degrading or inhibiting the translation of target genes, and are involved in the regulation of diverse biological processes, including cell differentiation, growth, mobility and death. Notably, it was reported that miRNA families play an essential role in regulating ischemia-induced cardiac injury, including cardiomyocytes necrosis, apoptosis, autophagy and fibrosis and so on $[9,22,23]$. MiR-124 is predominantly expressed in normal brain and plays an important role in neural processes from normal neural cell function to the development of central nervous system tumors [24]. Several studies have suggested that miR-124 is significantly downregulated in several human cancers, such as bladder, breast and lung cancer [25-27]. Recently, a number of experiments have shown that miR-124 is involved in the regulation of cardiovascular diseases. For example, the plasma expression of miR-124 is highly upregulated in patients with stable compensated dilated cardiomyopathy, indicating miR-124 may serve as a novel biomarker for diastolic dysfunction [28]. In addition, miR-124 was shown downregulated in myogenic conversion of bone marrow derived mesenchymal stem cells and affect the expression of cardiac-specific markers [17]. Furthermore, miR124 was elevated in AngII-induced hypertrophic cardiomyocytes and inhibition of miR-124 effectively suppressed myocardial hypertrophy [18]. However, the relationship between miR-124 function and myocardial infarction remains unclear.

In the present study, MI caused a significant increase of miR-124 level in the border and infarct areas of the myocardium as compared with that in the remote non-infarct area of the myocardium (Fig. 1). Meanwhile, the expression level of miR-124 is also significantly elevated in $\mathrm{H}_{2} \mathrm{O}_{2}$-stimulated cardiomyocytes, which is consistent with the in vivo results. These findings suggest that the elevation of miR-124 may contribute to the pathogenesis of MI. The mechanisms underlying MI injury is multifactorial, and apoptosis is one of the most important mechanisms. Recent studies have shown that inhibiting myocardial apoptosis 


\section{Cellular Physiology Cell Physiol Biochem 2018;51:186-200

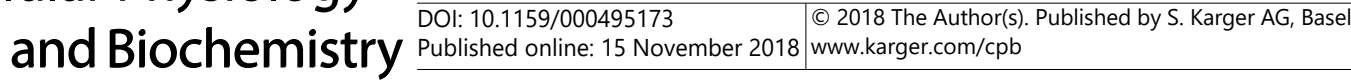 \\ He et al.: miR-124 Inhibition Reduces Cardiomyocyte Apoptosis}

will retard or even prevent heart failure induced by MI or ischemia/reperfusion injury [29]. Apoptosis can be regulated by the Bcl-2 protein family positively and negatively. It has been suggested that pro-apoptotic protein, Bax, translocation from the cytosol to mitochondria results in cytochrome c release from the mitochondria. While antiapoptotic protein, Bcl-2, can interfere with mitochondrial cytochrome $\mathrm{c}$ release and suppress apoptosis progression [30]. In this study, we found that silencing of miR-124 inhibited apoptosis in NRVMs, upregulated Bcl-2 expression and down-regulated Bax expression and caspase-3 activity. In addition, mitochondrial dysfunction is involved in myocardial MI injury [30]. And we tested the mitochondrial function in our study. The results indicate that the impaired mitochondrial $\Delta \psi$ m induced by $\mathrm{H}_{2} \mathrm{O}_{2}$ is improved due to AMO-124 pretreatment, suggesting that the mitochondrial dysfunction caused by oxidative stress was significantly attenuated. Moreover, our results suggest that knockdown of miR-124 following transfection with antagomir-124 improved the myocardial infarction and the injury of the cardiomyocytes, as indicated by improved cardiac function, reduced MI size and cardiac apoptosis, reversed the elevation of Bax/Bcl-2 ratio and stimulation of LDH and caspase- 3 activity. Our results indicate that inhibition of miR-124 can prevent the dysfunction of the mitochondria, leading to the reduction of following apoptosis, and protect against ischemic injury after MI.

Several studies have shown that miR-124 exhibits antitumor activity by directly suppressing STAT3 expression $[10,17]$. STAT3 has been demonstrated to be extensively expressed and also found to suppress cell apoptosis and induce tumor growth in various cell types [31]. It is able to activate multiple target genes, regulating numerous cellular functions at both the physiological and pathological level. STAT3 activation via phosphorylation on its Y705 residue stimulates gene expression affecting cell-cell adhesion, growth/ proliferation and has also been implicated during activation of anti-apoptotic Bcl proteins [32]. Furthermore, STAT3 activation has been demonstrated to provide cardioprotection from ischemic reperfusion injury [33]. Therefore, activation of STAT3 in the stressed heart is important for its protection of cardiomyocytes from specific apoptotic stimuli. The results of the present study indicate that knockdown of miR-124 inhibits the apoptotic potential of ischemic cardiomyocytes by upregulating STAT3. This conclusion is based on the following observations. First, TargetScan showed that the 3' UTR of STAT3 was directly targeted by miR-124. Second, the expression and activity of STAT3 were decreased in miR-124 silencing NRVMs but were elevated in miR-124 overexpressing NRVMs. Third, inhibition of miR-124 prevented the suppressed STAT3 activity in cardiomyocytes induced by ischemic injury. Finally, inhibiting STAT3 activity by Stattic eliminated the protective effect of miR-124 on enhanced cell viability and suppressed apoptosis of the NRVMs after $\mathrm{H}_{2} \mathrm{O}_{2}$ treatment. However, whether other signal pathways also contribute to the regulation of apoptosis of NRVMs by miR-124 need further investigation in the future.

\section{Conclusion}

In summary, our results demonstrate that knockdown of miR-124 exerts cardioprotection via preventing apoptosis and ameliorating mitochondrial dysfunction following MI by targeting STAT3. The findings suggest that miR-124 might be a potential therapeutic target for ischemic heart disease.

\section{Acknowledgements}

This work was supported in part by Major Program of National Natural Science Foundation of China (81230081). 


\section{Cellular Physiology Cell Physiol Biochem 2018;51:186-200 and Biochemistry \begin{tabular}{l|l} 
DOI: 10.1159/000495173 & (c) 2018 The Author(s). Published by S. Karger AG, Basel \\
www.karger.com/cpb
\end{tabular}

He et al.: miR-124 Inhibition Reduces Cardiomyocyte Apoptosis

\section{Disclosure Statement}

The authors declare no competing financial interests.

\section{References}

1 Li R, Yan G, Li Q Sun H, Hu Y, Sun J, Xu B: MicroRNA-145 protects cardiomyocytes against hydrogen peroxide $\left(\mathrm{H}_{2} \mathrm{O}_{2}\right)$-induced apoptosis through targeting the mitochondria apoptotic pathway. PLoS One 2012;7:e44907.

-2 Hu S, Huang M, Li Z, Jia F, Ghosh Z, Lijkwan MA, Fasanaro P, Sun N, Wang X, Martelli F, Robbins RC, Wu JC: MicroRNA-210 as a novel therapy for treatment of ischemic heart disease. Circulation 2010;122:S124-31.

- 3 Ren XP, Wu J, Wang X, Sartor MA, Jones K, Qian J, Nicolaou P, Pritchard TJ, Fan GC: MicroRNA-320 is involved in the regulation of cardiac ischemia/reperfusion injury by targeting heat-shock protein 20. Circulation 2009;119:2357-2366.

4 Sun C, Liu H, Guo J, Yu Y, Yang D, He F, Du Z: MicroRNA-98 negatively regulates myocardial infarctioninduced apoptosis by down-regulating Fas and caspase-3. Sci Rep 2017;7:7460.

5 Yang B, Lin H, Xiao J, Lu Y, Luo X, Li B, Zhang Y, Xu C, Bai Y, Wang H, Chen G, Wang Z: The muscle-specific microRNA miR-1 regulates cardiac arrhythmogenic potential by targeting GJA1 and KCNJ2. Nat Med 2007;13:486-491.

6 Bang C, Batkai S, Dangwal S, Gupta SK, Foinquinos A, Holzmann A, Just A, Remke J, Zimmer K, Zeug A, Ponimaskin E, Schmiedl A, Yin X, Mayr M, Halder R, Fischer A, Engelhardt S, Wei Y, Schober A, Fiedler J, Thum T: Cardiac fibroblast-derived microRNA passenger strand-enriched exosomes mediate cardiomyocyte hypertrophy. J Clin Invest 2014;124:2136-2146.

7 Tuttolomondo A, Simonetta I, Pinto A: MicroRNA and receptor mediated signaling pathways as potential therapeutic targets in heart failure. Expert Opin Ther Targets 2016;20:1287-1300.

-8 Recchioni R, Marcheselli F, Olivieri F, Ricci S, Procopio AD, Antonicelli R: Conventional and novel diagnostic biomarkers of acute myocardial infarction: a promising role for circulating microRNAs. Biomarkers 2013;18:547-558.

-9 Gupta SK, Foinquinos A, Thum S, Remke J, Zimmer K, Bauters C, de Groote P, Boon RA, de Windt LJ, Preissl S, Hein L, Batkai S, Pinet F, Thum T: Preclinical Development of a MicroRNA-Based Therapy for Elderly Patients With Myocardial Infarction. J Am Coll Cardiol 2016;68:1557-1571.

10 Li X, Yu Z, Li Y, Liu S, Gao C, Hou X, Yao R, Cui L: The tumor suppressor miR-124 inhibits cell proliferation by targeting STAT3 and functions as a prognostic marker for postoperative NSCLC patients. Int J Oncol 2015;46:798-808.

11 Xia J, Wu Z, Yu C, He W, Zheng H, He Y, Jian W, Chen L, Zhang L, Li W: miR-124 inhibits cell proliferation in gastric cancer through down-regulation of SPHK1. J Pathol 2012;227:470-480.

12 Furuta M, Kozaki KI, Tanaka S, Arii S, Imoto I, Inazawa J: miR-124 and miR-203 are epigenetically silenced tumor-suppressive microRNAs in hepatocellular carcinoma. Carcinogenesis 2010;31:766-776.

13 Xiao YT, Wang J, Lu W, Cao Y, Cai W: Downregulated expression of microRNA-124 in pediatric intestinal failure patients modulates macrophages activation by inhibiting STAT3 and AChE. Cell Death Dis 2016; 7:e2521.

14 Liu S, Song L, Zeng S, Zhang L: MALAT1-miR-124-RBG2 axis is involved in growth and invasion of HR-HPVpositive cervical cancer cells. Tumour Biol 2016;37:633-640.

15 Li Y, Zhang Z, Liu X, Huang T, He W, Shen Y, Liu X, Hong K, Cao Q: miR-124 functions as a tumor suppressor in the endometrial carcinoma cell line HEC-1B partly by suppressing STAT3. Mol Cell Biochem 2014;388:219-231.

16 Kerek R, Geoffroy A, Bison A, Martin N, Akchiche N, Pourie G, Helle D, Gueant JL, Bossenmeyer-Pourie C, Daval JL: Early methyl donor deficiency may induce persistent brain defects by reducing Stat3 signaling targeted by miR-124. Cell Death Dis 2013;4:e755.

17 Cai B, Li J, Wang J, Luo X, Ai J, Liu Y, Wang N, Liang H, Zhang M, Chen N, Wang G, Xing S, Zhou X, Yang B, Wang X, Lu Y: microRNA-124 regulates cardiomyocyte differentiation of bone marrow-derived mesenchymal stem cells via targeting STAT3 signaling. Stem Cells 2012;30:1746-1755. 


\section{Cellular Physiology Cell Physiol Biochem 2018;51:186-200 and Biochemistry \begin{tabular}{l|l|l|l}
\hline DOI: 10.1159/000495173 2018 The Author(s). Published by S. Karger AG, Basel & (c)
\end{tabular}

18 Bao Q, Chen L, Li J, Zhao M, Wu S, Wu W, Liu X: Role of microRNA-124 in cardiomyocyte hypertrophy inducedby angiotensin II. Cell Mol Biol (Noisy-le-grand) 2017;63:23-27.

19 Matsuda T ZP, Sciarretta S, Zhang Y, Jeong JI, Ikeda S, Park J, Hsu CP, Tian B, Pan D, Sadoshima J, Del Re DP: NF2 Activates Hippo Signaling and Promotes Ischemia/Reperfusion Injury in the Heart. Circ Res 2016;119:596-606.

20 Zhang JY GY, Si JP, Sun XB, Sun GB, Liu JJ: A polysaccharide of Dendrobium officinale ameliorates H2O2induced apoptosis in H9c2 cardiomyocytes via PI3K/AKT and MAPK pathways. Int J Biol Macromol 2017;104:1-10.

-21 Lu Y, Zhou J, Xu C, Lin H, Xiao J, Wang Z, Yang B: JAK/STAT and PI3K/AKT pathways form a mutual transactivation loop and afford resistance to oxidative stress-induced apoptosis in cardiomyocytes. Cell Physiol Biochem 2008;21:305-314.

22 Wang JX, Zhang XJ, Li Q Wang K, Wang Y, Jiao JQ Feng C, Teng S, Zhou LY, Gong Y, Zhou ZX, Liu J, Wang JL, Li PF: MicroRNA-103/107 Regulate Programmed Necrosis and Myocardial Ischemia/Reperfusion Injury Through Targeting FADD. Circ Res 2015;117:352-363.

23 van Rooij E, Sutherland LB, Thatcher JE, DiMaio JM, Naseem RH, Marshall WS, Hill JA, Olson EN: Dysregulation of microRNAs after myocardial infarction reveals a role of miR-29 in cardiac fibrosis. Proc Natl Acad Sci U S A 2008;105:13027-13032.

24 Sun Y, Luo ZM, Guo XM, Su DF, Liu X: An updated role of microRNA-124 in central nervous system disorders: a review. Front Cell Neurosci 2015;9:193.

25 Wang X, Wu Q, Xu B, Wang P, Fan W, Cai Y, Gu X, Meng F: MiR-124 exerts tumor suppressive functions on the cell proliferation, motility and angiogenesis of bladder cancer by fine-tuning UHRF1. Febs $\mathrm{j}$ 2015;282:4376-4388.

-26 Li L, Luo J, Wang B, Wang D, Xie X, Yuan L, Guo J, Xi S, Gao J, Lin X, Kong Y, Xu X, Tang H, Xie X, Liu M: Microrna-124 targets flotillin-1 to regulate proliferation and migration in breast cancer. Mol Cancer 2013;12:163.

27 Ma T, Zhao Y, Wei K, Yao G, Pan C, Liu B, Xia Y, He Z, Qi X, Li Z, Wang J, Shao Y: MicroRNA-124 Functions as a Tumor Suppressor by Regulating CDH2 and Epithelial-Mesenchymal Transition in Non-Small Cell Lung Cancer. Cell Physiol Biochem 2016;38:1563-1574.

28 Nair N, Kumar S, Gongora E, Gupta S: Circulating miRNA as novel markers for diastolic dysfunction. Mol Cell Biochem 2013;376:33-40.

29 Song XJ, Yang CY, Liu B, Wei Q, Korkor MT, Liu JY, Yang P: Atorvastatin inhibits myocardial cell apoptosis in a rat model with post-myocardial infarction heart failure by downregulating ER stress response. Int J Med Sci 2011;8:564-572.

-30 Korsmeyer SJ, Shutter JR, Veis DJ, Merry DE, Oltvai ZN: Bcl-2/Bax: a rheostat that regulates an anti-oxidant pathway and cell death. Semin Cancer Biol 1993;4:327-332.

31 Catlett-Falcone R, Landowski TH, Oshiro MM, Turkson J, Levitzki A, Savino R, Ciliberto G, Moscinski L, Fernandez-Luna JL, Nunez G, Dalton WS, Jove R: Constitutive activation of Stat3 signaling confers resistance to apoptosis in human U266 myeloma cells. Immunity 1999;10:105-115.

-32 Calo V, Migliavacca M, Bazan V, Macaluso M, Buscemi M, Gebbia N, Russo A: STAT proteins: from normal control of cellular events to tumorigenesis. J Cell Physiol 2003;197:157-168.

-33 Oshima Y, Fujio Y, Nakanishi T, Itoh N, Yamamoto Y, Negoro S, Tanaka K, Kishimoto T, Kawase I, Azuma J: STAT3 mediates cardioprotection against ischemia/reperfusion injury through metallothionein induction in the heart. Cardiovasc Res 2005;65:428-435. 